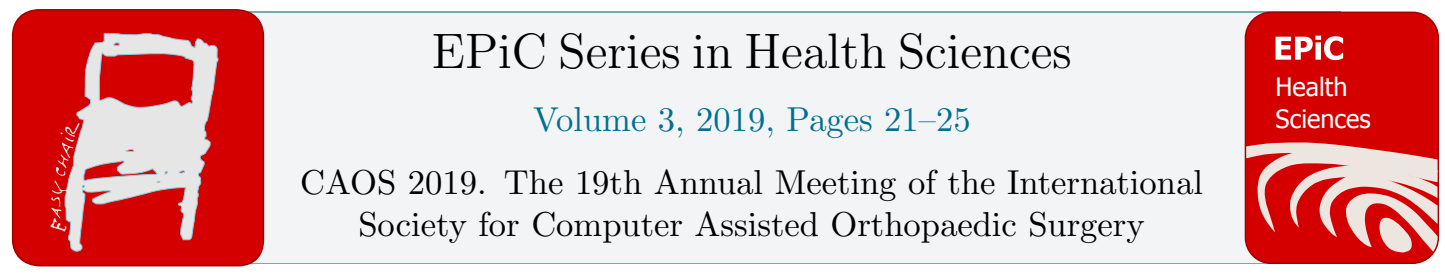

\title{
Automatic standardized shape analysis of the sagittal profiles (J-Curves) of the femoral condyles based on three-dimensional (3D) surface data
}

\author{
Malte Asseln ${ }^{1 *}$, Maximilian C. M. Fischer ${ }^{1}$, Hao Yang Chan $^{2}$, Patrick \\ Meere $^{2}$, Peter Walker ${ }^{2}$, Klaus Radermacher ${ }^{1}$ \\ ${ }^{1 *}$ Chair of Medical Engineering, Helmholtz Institute for Biomedical Engineering, RWTH Aachen \\ University, Aachen, 52074, Germany \\ ${ }^{2}$ Department of Orthopaedic Surgery, New York University Hospital for Joint Diseases, New \\ York, 10003, USA \\ asseln@hia.rwth-aachen.de
}

\section{Introduction}

The sagittal geometry of the articular surfaces of the femoral condyles, also called J-Curves because of the letter J-shaped profiles, is one of the main factors affecting knee kinematics in the normal knee [1] as well as artificial knee [2]. For example, Clary et al. [2] showed that large changes in the J-curves' radii cause abrupt changes in the center of rotation, leading to decreased anterior-posterior stability. In literature, the sagittal profile has been described mathematically by different geometric figures, such as arcs, circles, involutes of a circle, and Archimedean and logarithmic spirals [3]. The circular approximation has been often followed in the different concepts of knee implant designs, such as singleradius-, dual-radius-, or multiple-radius-designs. Single-radius-designs have a fixed flexion-extension axis. Dual-radius-designs consist of a larger distal and smaller posterior radius aiming a higher congruence during low flexion (high loading) and lower congruence at high flexion angles (high mobility). Multi-radius-designs try to mimic a physiological roll-glide ratio. However, the description of these circles is usually not standardized. A summary of different measurement methods was given by Nuno and Ahmed [4].

Thereby, the radii are very sensitive regarding the length of the fitting arc [5] and position of the sagittal plane [3]. Nuno and Ahmed [3] found that medial and lateral condyles can be adequately described by two-circular arcs and proposed a quantitative description. However, the posterior limits of their arcs were not considered individually, the anterior limits were defined based on soft-tissue measurements (anterior margins of the menisci), and the sagittal plane was positioned at the posterior extreme points, which might be inadequate in arthritic knees. 
The goal of this study was to automatically analyse the medial and lateral sagittal profiles of the femoral condyles mathematically by two-circular arcs in a standardized and robust fashion.

\section{Materials and Methods}

The study was based on bony femoral geometries of 20 male subjects (mean age: 26 years, range 18-40 years, mean BMI: 26), who had a prior anterior cruciate ligament repair 8-20 months previously. However, the actual geometries were considered as being normal [6]. All datasets were acquired from magnet resonance imaging, segmented manually, post-processed, and stored as polygon mesh (Figure $1(\mathrm{~A})$ ).

At first, a standardized anatomical coordinate system was calculated. A unified sagittal plane was iteratively calculated following $\mathrm{Li}$ et al. [5]. Ellipses were fitted in sagittal cuts and the connection of the posterior foci approximated a posterior cylindrical axis similar to the one described by Eckhoff et al. [7]. The plane normal was defined as $\mathrm{x}$-axis (medial/lateral) and used to calculate the femoral epicondyles. The $\mathrm{y}$-axis (anterior/posterior) was calculated as cross product of the $\mathrm{x}$-axis and the longitudinal body axis. The z-axis (proximal/distal) was defined as cross product of the $\mathrm{x}$-and $\mathrm{y}$-axis. The origin was located at the midpoint of the epicondyles.

The intersections of the posterior cylindrical axis and femoral geometry were calculated resulting in four reference points. The midpoints of the respective outer and inner points of each condyle were used to locate the sagittal cuts. Hence, they were positioned in the center of each condyle without considering the posterior extrema (Figure 1 (B)).

Subsequently, the two-dimensional (2D) intersections of the planes and bony femoral geometry were analysed following $\mathrm{Li}$ et al. [5]. A curvature analysis revealed the boundary points of the articulating portion. The posterior extremity and anterior extremity of the articulating surface were identified as points with the largest curvature values in respective regions. Finally, the circle leastsquare method proposed by Nuno and Ahmed [3] was applied resulting in a posterior and distal circular arc with tangential transition and continuity (Figure $1(\mathrm{C})+(\mathrm{D})$ ). The framework was implemented in MATLAB (The MathWorks, Inc., USA). Finally, the geometric parameters were statistically analysed and reported. 
(A)

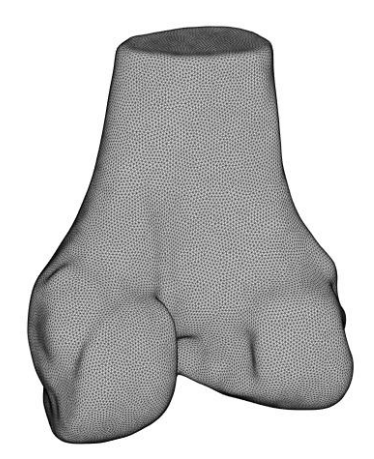

(C)

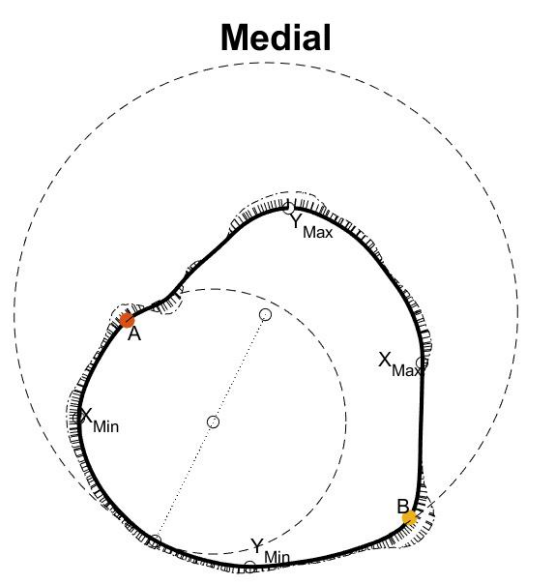

(B)

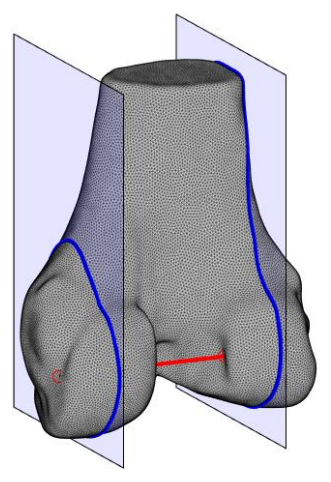

(D)

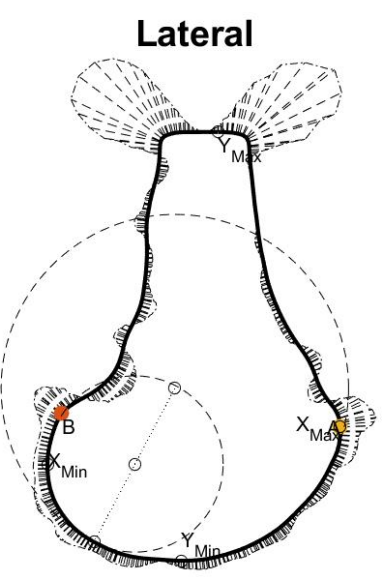

Figure 1: Exemplarily visualization of a femoral geometry (A), calculated posterior cylindrical axis (red), cutting planes, and intersections (B), medial intersections with two-circular arcs, curvature vectors (whiskers), and reference points (C), and lateral intersections with two-circular arcs (D).

\section{Results}

The framework was able to process all datasets without any computational error. The visual inspection revealed an accurate circular fitting. The statistical values are summarized in Table 1 . The mean medial and lateral posterior radii were approximately equal $(20.27 \pm 1.69 \mathrm{~mm}$ vs. $20.02 \pm 1.93 \mathrm{~mm})$. The mean lateral distal radius was slightly larger than medial $(42.47 \pm 5.63 \mathrm{~mm}$ vs. $39.42 \pm 7.60 \mathrm{~mm}$ ). However, the standard deviations were higher compared to the posterior radii. The posterior angle was similar for the medial and lateral condyle and in the range between approximately $31-59^{\circ}$. 


\begin{tabular}{lrrrrrrrrrr}
\hline & \multicolumn{1}{c}{ Medial } & \multicolumn{1}{c}{ Lateral } \\
& \multicolumn{1}{c}{ r_p } & r_d & alpha_p & alpha_1 & alpha_2 & r_p & r_d & alpha_p & alpha_1 & alpha_2 \\
\hline Mean & 20.27 & 39.42 & 45.75 & 25.92 & 44.98 & 20.02 & 42.47 & 44.61 & 22.38 & 57.31 \\
SD & 1.69 & 7.60 & 7.38 & 11.03 & 15.88 & 1.93 & 5.63 & 5.91 & 9.28 & 16.09 \\
Min & 16.29 & 28.27 & 30.98 & 6.20 & 6.51 & 17.05 & 34.09 & 32.28 & 0.50 & 24.11 \\
Max & 23.09 & 63.20 & 59.15 & 45.55 & 76.76 & 23.24 & 57.60 & 52.47 & 44.64 & 77.50 \\
\hline
\end{tabular}

Table 1: Statistical results of the geometric parameters for the medial and lateral compartment

\section{Discussion}

We presented a standardized approach to describe the medial and lateral sagittal profiles of the femoral condyles mathematically by two-circular arcs. This was basically achieved by the combination of two algorithms presented previously by Li et al. [5] and Nuno and Ahmed [3].

The results of the radii were in good agreement with the reported values. Nuno and Ahmed [3] reported $18.9 \pm 2.3 \mathrm{~mm}$ and $19.6 \pm 2.5 \mathrm{~mm}$ for the medial and lateral posterior radii, respectively. We found slightly larger values of $20.27 \pm 1.69 \mathrm{~mm}$ vs. $20.02 \pm 1.93 \mathrm{~mm}$. However, their study cohort included two female donors and ten male donors, whereas we considered male only. In addition, they used a fixed posterior angle of $30^{\circ}$ over all subjects. We found posterior angles in the range of $31-59^{\circ}$ demonstrating the individual variability and indicating that a constant angle is rather unsuitable. Looking at the distal radii, Nuno and Ahmed [3] reported values of $35.0 \pm 4.1 \mathrm{~mm}$ and $36.6 \pm 6.1 \mathrm{~mm}$, respectively. In comparison, our values were again higher, but confirmed the same tendency of a larger lateral distal radius.

In conclusion, the developed framework is capable of analysing fully automatic the medial and lateral sagittal profiles robustly in a standardized manner, solely based on femoral cutting profiles. Thus, the approach may also be easily transferred to standardized 2D lateral radiographs. The framework may also have the potential in femoral component design optimization and automatic morpho-functional studies of large data bases.

\section{References}

[1] Freeman MAR, Pinskerova V. The movement of the normal tibio-femoral joint. Journal of biomechanics 2005;38:197-208.

[2] Clary CW, Fitzpatrick CK, Maletsky LP, Rullkoetter PJ. The influence of total knee arthroplasty geometry on mid-flexion stability: an experimental and finite element study. Journal of biomechanics 2013;46:1351-7.

[3] Nuño N, Ahmed AM. Sagittal profile of the femoral condyles and its application to femorotibial contact analysis. Journal of biomechanical engineering 2001;123:18-26.

[4] Nuño N, Ahmed AM. Three-dimensional morphometry of the femoral condyles. Clinical biomechanics (Bristol, Avon) 2003;18:924-32.

[5] Li K, Tashman S, Fu F, Harner C, Zhang X. Automating analyses of the distal femur articular geometry based on three-dimensional surface data. Annals of biomedical engineering 2010;38:2928-36. 
[6] Chan HY, Walker PS, Lerner A, Chaudhary M, Bosco JA. Design of Reverse Materials Resurfacing Implants for Mild-Moderate Medial Osteoarthritis of the Knee. J. Med. Devices 2017;11:11004.

[7] Eckhoff D, Hogan C, DiMatteo L, Robinson M, Bach J. Difference between the epicondylar and cylindrical axis of the knee. Clinical orthopaedics and related research 2007;461:238-44. 